La comète a l'aspect d'une nébulosité à bords très diffus, d'environ $15^{\prime \prime}$ de diamètre, avec condensation un peu excentrée vers l'Est et peut être un très petit noyau; l'éclat total est de $10 \cdot 5$. - Les 5 et 6 , images très agitées; la

St. Genis-Laval (Rhône), 1906 mars 9. comète est difficile à pointer pendant la deuxième série. Le 7, observation difficile à cause de la Lune; la comète paraît une demi grandeur plus faible que l'étoile de comparaison qui est de $\mathrm{II}^{\mathrm{m}}$. - Le 8 , comète invisible, à cause de la Lune.

\title{
Neue Elemente und Ephemeride des Kometen 1906 b.
}

Die folgenden parabolischen Elemente sind abgeleitet aus den Beobachtungen 1906 März 3 (Kopf), März 9 (Javelle), März I 7 (Rheden).

Der mittlere Ort wird genau dargestellt.*)
F. Guillaume.

Ephemeride für I $^{\text {h }}$ M. Z. Berlin.

\begin{tabular}{|c|c|c|c|c|c|c|c|c|c|c|c|}
\hline 1906 & $\alpha$ vera & $\delta$ vera & $\log r$ & $\log \Delta$ & $\mathrm{H}$ & I 906 & $\alpha$ vera & $d$ vera & $\log r$ & $\log A$ & $\mathrm{H}$ \\
\hline lärz 17 & $I_{1}^{\mathrm{h}} 29^{\mathrm{m}} 57^{\mathrm{s}}$ & $+2^{0} \quad 0^{\prime} 2$ & 0.5256 & $0.373^{\circ}$ & 0.94 & April Io & I $\mathbf{I}^{\mathrm{h}} 2 \mathbf{I}^{\mathrm{m}} 59^{\mathrm{s}}$ & $+2^{\circ} 26: 7$ & 0.5376 & 0.4053 & 0.78 \\
\hline 19 & 29 & 3.2 & & & & I 2 & 2137 & $\begin{array}{ll}2 & 27.4\end{array}$ & & & \\
\hline 21 & $28 \quad 18$ & 26.1 & 0.5275 & 0.3766 & 0.92 & $x_{4}$ & 2 I 18 & $2 \quad 27.9$ & 0.5397 & 0.4128 & 0.74 \\
\hline 23 & 2730 & 29.0 & & & & 16 & 2 I 3 & $2 \quad 28$ & & & \\
\hline 25 & $26 \quad 43$ & $\begin{array}{lll}2 & 1 & 1.7\end{array}$ & 0.5294 & 0.3810 & 0.89 & I 8 & $205 \mathrm{I}$ & $2 \quad 27.9$ & 0.5418 & 0.4208 & 0.70 \\
\hline 27 & $25 \quad 59$ & $2 \quad 14.3$ & & & & 20 & 2043 & $\begin{array}{ll}2 & 27.4\end{array}$ & & & \\
\hline 29 & 2516 & $2 \quad 16.7$ & $0.53 \times 4$ & $0.386 \mathrm{x}$ & 0.87 & 22 & 2038 & $2 \quad 26.5$ & $0.544^{\circ}$ & 0.4291 & 0.67 \\
\hline $3 I$ & 2436 & $=19.0$ & & & & 24 & 2038 & $2 \quad 25.4$ & & & \\
\hline April 2 & 2359 & 221.0 & 0.5334 & 0.3919 & 0.83 & 26 & 2040 & $\begin{array}{ll}2 & 23.9\end{array}$ & 0.5462 & 0.4378 & 0.64 \\
\hline 4 & 2325 & $2 \quad 22.8$ & & & & 28 & 2047 & 222.0 & & & \\
\hline 6 & 2253 & $2 \quad 24.4$ & 0.5355 & 0.3983 & $0.8 \mathrm{I}$ & 30 & $205^{8}$ & 220.0 & 0.5484 & 0.4468 & $0.6 \mathrm{I}$ \\
\hline 8 & $22 \quad 24$ & $2 \quad 25.7$ & & & & Mai & 2 I 12 & $2 \quad 17 \cdot 3$ & & & \\
\hline 10 & $\begin{array}{lll}111 & 21 & 59\end{array}$ & +226.7 & $0.537^{6}$ & 0.4053 & 0.78 & 4 & I I 2 I 30 & $+2 \div 4.4$ & 0.5506 & 0.4560 & 0.57 \\
\hline
\end{tabular}

Einheit der Helligkeit 1906 März 3.

Kiel, Bureau der Astron. Nachr., I 906 März 2 I.

M. Ebell.

*) Die an sich naheliegende Vermutung, daß der Komet za den kurzperiodischen gehöre, wird bisher durch die Beobachtungen nicht bestätigt. Ein vor der Berechnung der Parabel unternommener Versuch, aus denselben Beobachtungen einen Kegelschnitt ohne Voraussetzung der Exzentrizität abzuleiten, ergab zwar eine Ellipse, aber mit einer nur als Rechnungsresultat anzusehenden Umlaufszeit von I I53.5 Jahren.

Beobachtungen des Kometen $1906 \mathrm{~b}$.

\begin{tabular}{|c|c|c|c|c|c|c|c|c|c|c|c|}
\hline 1906 & M. Ortszeit & $\Delta \alpha$ & $\Delta \delta$ & Vgl. & $\mathrm{Bb}$. & $\alpha$ app. & $\log p \cdot \Delta$ & $\delta$ app. & $\log p .4$ & Red. ad 1. app. & * \\
\hline
\end{tabular}

Auf der Sternwarte des Collegio Romano in Rom.

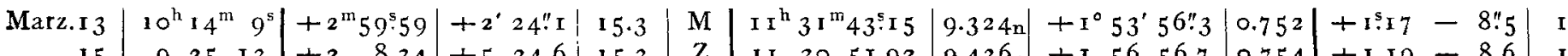

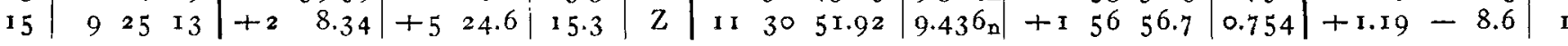

$\mathrm{M}=E$. Millosevich, $\mathrm{Z}=$ G. Zappa. - Marzo I3. Nucleo stellare di $10^{\mathrm{m}}{ }_{5}$, debolissima nebulosità.

Am $325 \mathrm{~mm}$ Refraktor des astronom. Instituts Königstuhl-Heidelberg von Hofrat W. Valentiner.

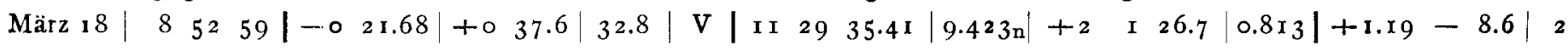

A uf der Sternwarte in Straßburg von Dr. C. W. Wirtz.

März $17 \quad 1^{\mathrm{h}} 40^{\mathrm{mm}} 3^{\mathrm{s}}$ M. Z. Straßburg $\alpha(1906.0)=\operatorname{II}^{\mathrm{h}} 29^{\mathrm{m}} 57^{\mathrm{s}} .56 \quad \delta(1906.0)=+2^{\circ} 0^{\prime}$ I 2."5

Unbestimmt gestaltete Nebelhülle mit Kern I $_{1}{ }^{\mathrm{m}}{ }_{5}$, Totaleindruck ${ }_{1}{ }^{\mathrm{m}}$, Durchm. $=0^{\prime} 7$. Die Nebelmaterie scheint sich im PW. $270^{\circ}$ etwas weiter auszudehnen (Schweif?).

Auf der k. k. Sternwarte in Wien von Dr. F. Rheden (Telegramm).

März $7 \quad \mathbf{I}^{\mathrm{h}} 27^{\mathrm{m}} \cdot 5$ M. Z. Wien $\alpha$ app. $=172^{\circ} 29^{\prime} \mathrm{I} 9^{\prime \prime} \delta$ app. $=+2^{\circ} \circ^{\prime} 9^{\prime \prime} \quad$ Gr. $\mathrm{II}^{\mathrm{m}}$. 